\title{
Inherited retinal disorders in South Africa and the clinical impact of evolving technologies
}

\author{
L Roberts, ${ }^{1}$ MSc; R Goliath, ${ }^{1} \mathrm{PhD}$; G Rebello, ${ }^{1} \mathrm{PhD} ; \mathbf{S}$ Bardien, ${ }^{1,3} \mathrm{PhD} ;$ A V September, ${ }^{1,2} \mathrm{MSc}$, PhD; L Bartmann, ${ }^{1} \mathrm{RN} ; \mathrm{F}$ Loubser, ${ }^{1}$ MSc; \\ L J Greenberg, ${ }^{1} \mathrm{PhD}$; R S Ramesar, ${ }^{1} \mathrm{MSc}, \mathrm{PhD}$ \\ 'Division of Human Genetics, Department of Pathology, Institute of Infectious Disease and Molecular Medicine, Faculty of Health Sciences, \\ University of Cape Town, South Africa \\ ${ }^{2}$ Division of Exercise Science and Sports Medicine, Department of Human Biology, Faculty of Health Sciences, University of Cape Town, South Africa \\ ${ }^{3}$ Division of Molecular Biology and Human Genetics, Faculty of Medicine and Health Sciences, Stellenbosch University, Tygerberg, South Africa
}

Corresponding author: L Roberts (lisa.roberts@uct.ac.za)

\begin{abstract}
Retinal degenerative disorders (RDDs) encompass a group of inherited diseases characterised by vision loss. The genetic and clinical complexity poses a challenge in unravelling the molecular genetic aetiology of this group of disorders. Furthermore, the population diversity in South Africa (SA) presents researchers with a particularly complicated task. Rapid advances in the development of cutting-edge technological platforms over the past two decades, however, have assisted in overcoming some of the challenges. The RDD research team has utilised these escalating technologies, which has facilitated a corresponding increase in molecular diagnoses. A biorepository has been established and comprises 3 200 patient DNA samples archived with many forms of RDD (including retinitis pigmentosa, macular dystrophies, Stargardt disease, Leber congenital amaurosis, Usher syndrome and Bardet Biedl syndrome). A comprehensive review is presented of the SA journey spanning 25 years, into elucidating the molecular genetic basis of various forms of RDD in SA.
\end{abstract}

S Afr Med J 2016;106(6 Suppl 1):S33-S37. DOI:10.7196/SAMJ.2016.v106i6.10988

The massive strides made in human genetics in the last 10 years would not have been possible without the diligent and insightful work of the pioneers in human genetics. This is particularly true in South Africa (SA), where a small community of scientists and clinicians have been responsible for the application of scientific/ medical advances into the wider SA population. Prof. Peter Beighton spearheaded the initial retinal degenerative disorder (RDD) research project in 1985, the emphasis being the identification of genes underlying RDD in SA families.

The RDD genes affect the rod and cone photoreceptors of the retina, resulting in retinal degeneration and consequent loss of vision, which may result in total blindness. At the time the project was initiated, it was believed there were, at most, three genes responsible for the most common RDD, namely retinitis pigmentosa (RP): one each for the autosomal dominant (ADRP), the autosomal recessive (ARRP) and the X-linked inherited (XLRP) forms. To date, however, there are 21, 34 and 2 genes for these forms of RP, respectively, ${ }^{[1]}$ and over 230 genes for all forms of RDD worldwide.

Generally, inherited RDDs are a challenging group of disorders to characterise, as they display vast clinical and genetic heterogeneity. Nevertheless, providing a genetic diagnosis to patients affords several benefits, including predictive, carrier and diagnostic testing for family members and participation in current and future gene-based clinical trials.

Genetics in SA has its own unique challenges; the populations, both local and immigrant, exhibit genetic diversity of a higher order than other population groups. ${ }^{[2]}$ Some groups have remained genetically isolated from each other, while others exhibit significant admixture. These phenomena have collectively contributed to unique, local variations in gene frequencies, noted in all SA population groups, which has further confounded genetic diagnosis for RDDs. Nevertheless, 'gene-hunting' for this group of disorders in SA has changed dramatically over the 25 years since the project began, largely through evolving molecular technologies, together with the computational era and increasing bioinformatics capabilities. This article gives an overview of the research efforts in SA into RDD.

\section{The RDD biorepository and database in Human Genetics at the University of Cape Town (UCT)}

Successful gene-hunting relies on good family and phenotype data, and to this end an electronic database containing patient and family information was constructed. From the outset, the emphasis was not only on the collection of biological specimens (mostly blood) but that the accompanying data were of the highest standard in order to support meaningful genetic analysis. Sampling and pedigree collection were carried out by genetic nursing sisters who arranged for patients to visit Groote Schuur Hospital in order to confirm their diagnoses and to offer clinical management wherever possible.

I was ushered into the world of research regarding RDDs. The task was to liaise between affected families, the Retinal Preservation Foundation (currently Retina SA) and the then Department of Human Genetics at UCT. I collected blood samples from the families affected with RDDs and extended the families by building pedigrees, a challenge that took us back to our founding fathers. The blood clinics were carried out with the help of the Retina SA branches, nationally, and the genetic nurses from the various regional Department of National Health offices around the country. Between these organisations we built a very healthy relationship with the families, counselling and encouraging affected persons to push through, overcome their disability and to reach their dreams!' - LB. 


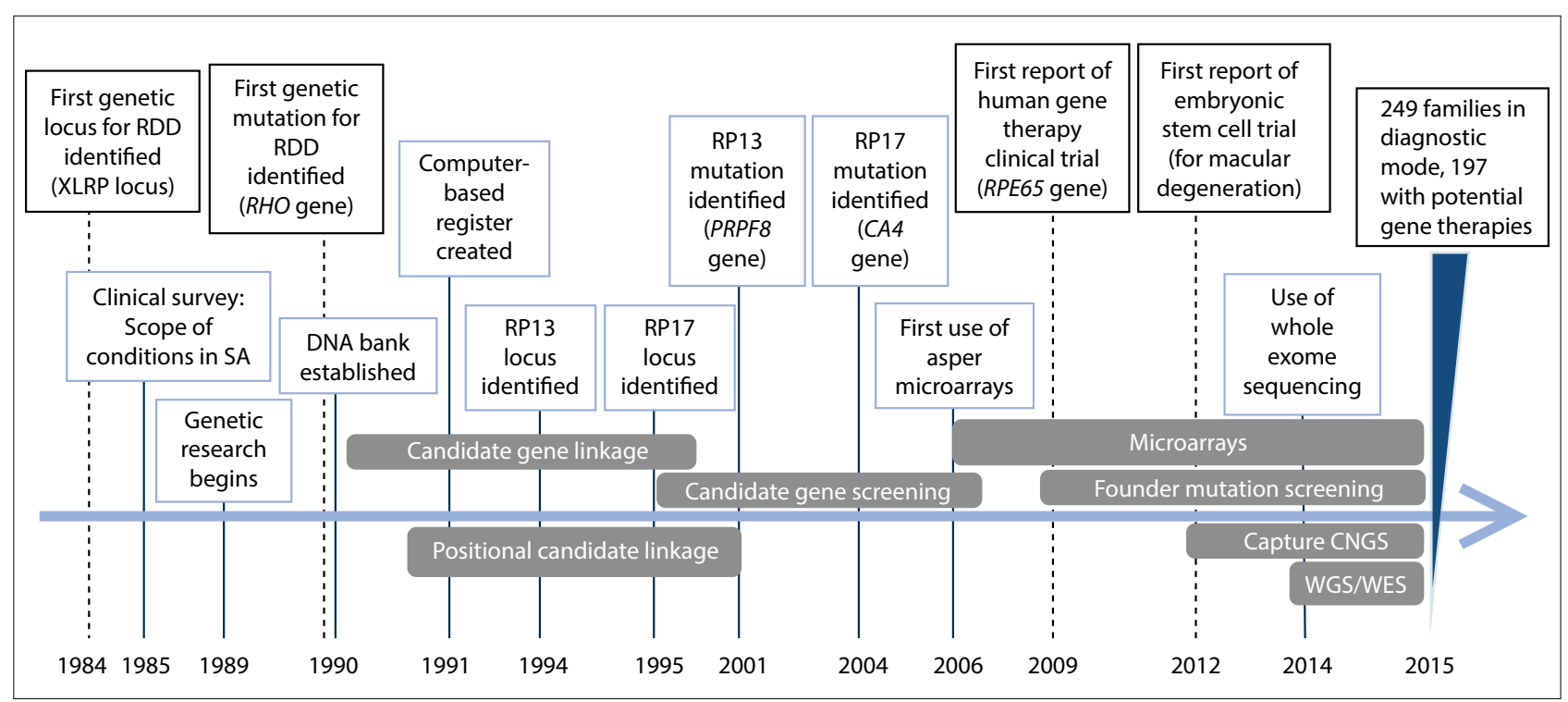

Fig. 1. Timeline of milestones reached during the 25 years of retinal research in SA. The black boxes represent international milestones, while the blue boxes represent local SA milestones. The molecular approaches used during different time periods are indicated in grey. (CNGS = capture next-generation sequencing; $W G S=$ whole genome sequencing; WES = whole exome sequencing.)

In 1985, a questionnaire-based survey was conducted with local SA families, in order to determine the scope of RDDs in the local population and whether they would benefit from advances in genetic screening. The first report on the findings of this initial study contained 63 families comprising 130 affected individuals. ${ }^{[3]}$ This initial cohort of families was the foundation upon which the biorepository was built. Five years later, in 1990, a DNA bank ${ }^{[4]}$ and computer-based register were established, and by 1995 the RDD team reported a cohort of 75 families, 440 individuals, which included 153 affected patients. ${ }^{[4]}$

The sample collection and analysis was ongoing and in 2002 Rebello et al..$^{[5]}$ reported a registry of information on 531 families of 1829 individuals (comprising 802 affected RDD patients). At this stage the database included the combined clinical, biological sample and research data on the entire cohort of samples. Interrogation of the database led to a report in 2003 by the UCT RDD research group that showed skewing of samples with strong underrepresentation of the indigenous SA ethnic groups. ${ }^{[6]}$ Recruiting efforts then focused on addressing the potential selection bias affecting the ethnic balance of the data and, more importantly, providing diagnoses and interventions to the previously disadvantaged communities in SA. As of August 2015, the registry contained 3237 individuals in 1430 families.

The biorepository remains, however, an incomplete portrait of RDD in SA, as indigenous Africans comprise only 20\%

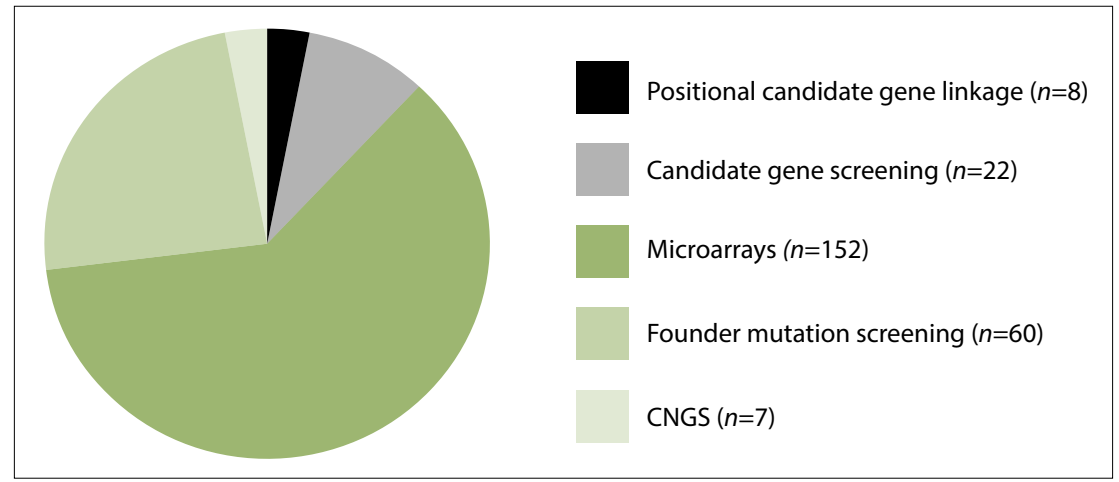

Fig. 2. The number of families in the RDD biorepository for whom a genetic diagnosis for RDD was determined, using various molecular technologies.

of samples, while this demographic group actually represents $79 \%{ }^{[7]}$ of the total population in the country. Additionally, information is available for 1314 individuals in SA who have been diagnosed with RDDs but from whom biological material has not yet been collected. There is therefore an ongoing drive to recruit indigenous SA families and expand the biorepository.

The biorepository is an invaluable resource facilitating the aims of $\mathrm{RDD}$ research endeavours in SA.

\section{Evolution of molecular genetic technologies}

The application of human molecular genetics as a tool to identify disease-causing mutations underpinning classic Mendelian inherited conditions of families have evolved over the decades. The traditional research methods of the 1970s through to the early 1990s primarily employed the hypothesis- driven 'candidate gene' linkage approach in an attempt to identify RDD genetic loci in large pedigrees. However, recent years have seen a dramatic shift in both the application of molecular genetics and the research methodologies used. This shift has come about through continued reviewing and updating of the research approach, underpinned by evolving molecular technologies. This dynamic approach has supported a significant increase in the rate of the emergence of translational research outputs. Fig. 1 gives an outline of RDD research milestones against the evolving approaches and technologies employed over the past 25 years. Fig. 2 outlines the number of families in whom disease-causing molecular defects were identified, using the various research approaches and technologies.

The initial approach used in the genehunting research endeavours of RDD SA was linkage analysis using microsatellite 
markers. These markers had been shown to be tightly linked to genes associated with RDD. Candidate gene analyses allowed exclusion of linkage from these genes and loci in the five largest SA RDD families. This was an important and exciting finding as it presupposed that there was possibly a unique molecular aetiology underlying RDD in SA.

The emergence of whole chromosome microsatellite marker maps enabled researchers to screen the genome for linkage analysis with (i) an unbiased and/or (ii) a positional candidate gene approach. The latter approach screened for linkage to genes whose products held either a structural or biochemical function which facilitate vision, but had not previously been implicated in RDD. The application of this approach led to the identification of linkage to markers on chromosome $17 \mathrm{p}$ in the largest RDD family in SA. ${ }^{[8]}$ This identification of the RP13 locus was the first major milestone of the research endeavours of RDD in SA. Furthermore, not only was it the first RDD locus identified in SA, but it was also a novel ADRP locus worldwide. Shortly thereafter in 1995, using the same approach in another SA RDD family, a second novel ADRP locus, RP17, was elucidated, mapping to chromosome $17 \mathrm{q} \cdot{ }^{[9]}$

Subsequent efforts to identify the specific RP13 and RP17 genes took 7 and 9 years, respectively. These undertakings involved fine mapping and screening of candidate genes in the critical chromosomal intervals at the two loci. Candidate genes were selected based on reported functions in the visual system. However, ultimately, the causative gene at the RP13 locus turned out to be an unlikely candidate - a transcription factor, $P R P F 8,{ }^{[10]}$ and $C A 4$ (a carbonic anhydrase enzyme) was identified as the $R P 17$ gene. ${ }^{[11]}$ This proves that the original gene-hunting methods did work, but had significant limitations as large families (with many affected and unaffected individuals and spanning multiple generations) were required for study.

Advances in the human genome project, ${ }^{[12]}$ the accompanying development of denser microsatellite marker maps and the characterisation of genes along long chromosomal stretches, facilitated more direct approaches to disease-gene hunting. This direct candidate gene screening approach involved the targeted analysis of coding regions and intron/exon boundaries of candidate genes that had been reported internationally for specific mutations. Cohorts of patients were selected for each RDD candidate gene screen, based on their phenotype (i.e. clinical diagnosis and mode of inheritance) and screened for mutations using single stranded conformational polymorphism analysis (SSCP), and later the higher throughput denaturing high performance liquid chromatography (dHPLC).

This involved analysing genes, portion by portion (less than $500 \mathrm{bp}$ at a time), in a single affected individual per family, to detect mutations. Although large families were not required for this candidate screening approach, analysis remained laborious and slow and was complicated by the fact that (i) unexpected causative genes that were not obvious candidates, e.g. ubiquitously-expressed splice factors and transcription factors had previously been associated with RDDs; (ii) there is vast clinical and genetic heterogeneity as well as significant genetic overlap between different RDDs. These scenarios make hypothesis-driven candidate gene selection problematic. Nevertheless, the ongoing research in the division has identified the causative mutation in several families over the years. ${ }^{[13]}$

The development of microarray technologies enabled escalation of screening to a level that allowed for whole genes to be analysed in large cohorts in a relatively short time period. In 2006, microarray technology (as offered by Asper Ophthalmics ${ }^{[14]}$ in Estonia) was incorporated into the RDD project. Each of 18 ophthalmic 'DNA chips' allow for the simultaneous screening for all reported mutations associated with a particular RDD. For example, the ADRP chip currently tests for 414 variants in 16 genes, and the ARRP chip currently tests for 710 variants in 28 genes. Using this approach, causative mutations are rapidly detected and founder mutations recognised and used to create targeted diagnostic tests for the specific population groups in which they were identified. An example is the test for seven mutations in $A B C A 4$ that are common in individuals of Afrikaner ancestry with Stargardt disease, ${ }^{[15]}$ and the test for a single mutation in MYO7A that is a frequent cause of Usher syndrome in indigenous Africans. ${ }^{[16]}$

A possible limitation of using the Asper Ophthalmic Microarrays is that the array platforms are designed to test for mutations reported globally, primarily based on data available from European and American individuals, and may not be relevant in the diverse population groups of SA. Furthermore, the arrayed primer extension (APEX) technology ${ }^{[17]}$ only sequences those select nucleotide positions in genes where mutations have been reported previously, therefore these chips are unable to detect novel mutations that are likely to exist in the unique SA population groups.

The growing need for an unbiased approach to gene-hunting, prompted the use of CNGS, for the first time on the RDD project in 2012. CNGS, as offered by the National Health Service in Manchester, $\mathrm{UK}^{[18]}$ enriches all the exons of a panel of target genes for sequencing. Initially the CNGS panel comprised the complete coding sequences of $105 \mathrm{RDD}$ genes, but currently it allows for the detection of novel and/or known variants by sequencing $176 \mathrm{RDD}$ genes. Due to current cost considerations, a limited number of samples have been tested with this approach $(n=11)$. However, it has proven successful and a molecular diagnosis was confirmed in seven families.

Taken together, the research approaches and technologies employed over the years have allowed the identification of the molecular cause of RDD disease in 249 families, for whom diagnostic testing is now available. The subsequent translational research efforts have seen results delivered to 416 individuals based on the work carried out in their families. Rewardingly (as of August 2015), 197 families are in a position to benefit from emerging gene therapy trials for the following genes: RPE65, AIPL1, MYO7A, ABCA4, and CHM.

The future direction for RDD research and gene hunting is to employ a true candidate-free approach: using NGS strategies such as WGS or WES. The strength of these approaches is that causative mutations can be identified without a pre-existing hypothesis of which genes to interrogate. This will allow for the detection of not only novel mutations in known genes, but novel genes are also likely to emerge ${ }^{[19]}$ resulting in a more comprehensive list of disease-causing mutations and the generation of potential key disease modulators. Compared with the original techniques, where one gene could take months or years to screen, capabilities in 2015 allow for sequencing of the entire exome (i.e. all coding portions of all 20000 - 30000 known human genes) in a matter of days, although the analysis is challenging and can take longer, particularly if the clinical information or family history is unclear.

\section{Phenotyping and the longitudinal study of famillies}

It is perhaps surprising that given the limited resources, manpower and 'archaic' labour-intensive technologies that were applied, we were able to molecularly diagnose 249 families with several retinal phenotypes. The successes can possibly be ascribed to the meticulous clinical profiling by the clinical geneticist, genetic nurses and allied professionals who laid the foundation for potential discoveries.

It can therefore be inferred that going forward, the limiting factor for new discoveries is perhaps not the invention of new 
"The ophthalmological fraternity was always

accommodating and filed many examination forms for

us. Included in these reports was accurate documentation of the state of the retina and vision of the patient. To

obtain these, several uncomfortable tests had to be undertaken, but it gave us a very accurate status of the disease for each individual.' - LB.

methodologies/technological platforms but rather the integrity of the clinical and biological material that is being researched. The documentation of the clinical affection status of family members is critical; one incorrect diagnosis, e.g. mislabelling of an affected individual as unaffected, would compromise the integrity of all downstream analyses. This is perhaps the single most important lesson to be remembered and applied as we traverse the landscape of the modern genomics era.

While every effort was made at keeping abreast with emerging technologies and research approaches, using First-World capabilities in a developing country such as SA, the over-arching goal was, and remains, the opportunity to offer any findings back to the patients and their interested families. ${ }^{[20]}$ With this in mind, the value of engaging fully with our research participants, ${ }^{[21]}$ eliciting extended family trees with a history of disease through the family and, where possible, attempting to trace the origin of the disease back to the countries from which the patients might have emerged, is an ongoing endeavour in the SA RDD research environment. The longitudinal progression of disease in SA RDD families was tracked over the last 25 years through working with a highly effective national network of ophthalmologists. This emphasis on patterns of heritability and ophthalmological course of disease is hugely important in terms of streamlining the potential gene candidates out of the very many involved in RDDs, thereby making research more effective.

An important aspect to consider is the complex genetic landscape of RDDs, which represents a significant challenge for identifying therapeutic targets and demonstrating proof-of-concept/s. This requires careful consideration of the genetic nature of the disease against the mutation prevalence, relative to disease severity and, importantly, the age of onset. Interrogation of family data is therefore an ongoing exercise.

\section{The real vallue to families}

What is embodied in the research of the RDD programme is a commitment to holistic care for individuals with inherited disorders. The identification of the causative mutation for an inherited RDD has immediate benefits for affected individuals, their families and their clinical management team. Establishing a genetic cause confirms the clinical diagnosis at a molecular level, enables accurate recurrence risk estimation, informed reproductive decision making as well as carrier testing or predictive testing (depending on the inheritance pattern and age of onset). Furthermore, knowledge of the specific gene involved may aid in prognosis, suggest potential therapeutic options and allows some patients to join clinical trials. The goal of medical research is therefore to aid in the genetic management of families, to alleviate disease symptoms through the development of treatment modalities, or to find a cure. In keeping with this ideology, the initial aim of the research project has been to identify the diseasecausing mutations in patients affected by inherited RDD and to translate the research findings into clinical practice and management. An important aspect of the SA RDD project has therefore always been the delivery of research findings to families. This undertaking engages a multidisciplinary team that includes medical scientists, medical geneticists, genetic counsellors, ophthalmologists, genetic nursing sisters, as well as the lay society, Retina South Africa, the registered non-profit and public-benefit lay organisation that has funded this research. Diagnostic services have been developed to provide a comprehensive clinical genetic service for RDD patients and their families in SA. The SA journey from 'DNA to diagnosis', and the subsequent translational research aspect of the project has, moreover, through the incorporation of genetic counsellors into the programme, highlighted their value in the uptake of findings and management of families.

With the capability of disease-gene and mutation identification, comes the capacity to manage further transmission of the disorder within the family through preimplantation genetic diagnosis (PGD), which is now available in SA. The process firstly involves in vitro fertilisation followed by preimplantation testing of the embryos. Embryos shown not to carry the known familial cause of RDD are then selected for implantation. Depending on the severity and age of onset within the family, some couples might consider prenatal diagnostic testing. The ethical and psychosocial issues pertaining to prenatal and preimplantation genetic diagnosis are complex and should be considered carefully through the process of genetic counselling. In 2015, a family with an X-linked form of RDD elected to utilise the PGD option that resulted in the birth of a healthy child who did not carry the disease-causing X-linked mutation segregating in the family. This case illustrates the essential role of clinical genetic services related to RDDs throughout SA over the past 25 years. The process started with a Cape Town couple from a SA RDD family, DNA has been archived from various family members from throughout SA and stored at UCT since 1999. The exact molecular genetic mutation was identified in 2007 in a laboratory in the UK, and in 2015, it was possible for the multidisciplinary team of professionals to facilitate PGD for this family with a positive and successful outcome.

\section{Disculssion}

Notwithstanding the unique challenges in the SA context, RDD research efforts over the past 25 years have enjoyed several major successes. The identification of novel genes, mutations and founder mutations in the SA RDD patient cohort has impacted the community, which this research serves, in an indelible manner. Furthermore, the research group has been very successful in its participation in the global RDD research arena. Group members' contribution to publications and their presentations at local and international scientific conferences are ongoing. Much of the success can be attributed to the insight and forethought of Prof. Peter Beighton and his response by getting involved in a variety of areas - he is probably one of the last 'polymaths' one will ever encounter. In his era, empiric risk calculation was based on more than just what the patient presented to the clinician, but also an understanding of how the disease affected other members of the family. Even then, there was the anticipation of one day finding the underlying gene - despite not having the technologies available.

Over the years, gene hunting approaches have shifted as technologies improved and became cost-effective. Every effort has been made to introduce new technologies into the research/training/ education environment. This is not only to ensure that trainees and postgraduates are exposed to state-of-the-art technologies, and trained in the applicability of such technologies, but also to ensure that research for the various disorders is performed by highly trained researchers in SA, for SA. Advances in molecular genetics (and genomics) have infiltrated almost all areas of biology and are currently employed as the research tools commonly used in a 
multidisciplinary environment to explore the molecular mechanisms for both common multifactorial diseases and rare Mendelian diseases. High-throughput technologies are mostly incorporating hypothesisfree approaches. Large multi-centre collaborations, exploring genetic and environmental contributions to disease, are common practice to try and unravel the missing heritability. However, the question remains: 'Have the traditional principles and practices of medical genetics as it relates to detailed clinical phenotyping and recording of medical and family history of the 1970s - 1990s been adopted in this new era of the genomics revolution?'

What is obvious is that despite the high-throughput technologies currently implemented in the laboratory at UCT, the practice of true geneticists, as embodied in Peter Beighton, will remain a gold standard. This involves the long-term engagement of families (and communities) where the research is seen as a jointly driven enterprise, committed to feeding back progress in research, as well as translating discoveries to patients, families and communities.

We salute Prof. Beighton for his remarkable wisdom, insight and tenacity, and for reminding us that at the heart of research, there is a patient and a family. This should be remembered as the next generation of scientists are trained in the 'next-generation' technologies.

\section{References}

1. RetNet: The Retinal Information Network. Summaries of genes and loci causing retinal diseases. Texas: University of Texas-Houston Health Science Center, 2016. https://sph.uth.edu/Retnet/sum-dis.html (accessed 25 August 2015).

2. Ramsay M, Tiemessen CT, Choudhury A, Soodyall H. Africa: The next frontier for human disease gene discovery? Hum Mol Genet 2011;20(R2):R214-R220. DOI:10.1093/hmg/ddr401
3. Oswald AH, Goldblatt J Sampson G, Clokie R, Beighton P. Retinitis pigmentosa in South Africa. S Afr Med J 1985;68(12):863-866

4. Greenberg J, Ramesar R, Beighton P. Genetic mapping of retinitis pigmentosa implications for Sout African patients. S Afr Med J 1994;84(7):410-412.

5. Rebello MT, Greenberg LJ, Ramesar RS. A computer-based register for inherited retinal dystrophies in Southern Africa. Ophthalmic Genet 2002;23(1):61-65.

Ramesar RS, Roberts L, Rebello G, et al. Retinal degenerative disorders in Southern Africa A molecular genetic approach. Adv Exp Med Biol 2003;533:35-40.

7. Statistics South Africa. Census 2011 Products: Census 2011 Fact Sheet. http://www.statssa.gov. za/?page_id $=3955$ (accessed 12 October 2015).

8. Greenberg J, Goliath R, Beighton P, Ramesar R. A new locus for autosomal dominant retinitis pigmentosa on the short arm of chromosome 17. Hum Mol Genet 1994;3(6):915-918.

Bardien S, Ebenezer N, Greenberg J, et al. An eighth locus for autosomal dominant retinitis pigmentosa is linked to chromosome 17q. Hum Mol Genet 1995;4(8):1459-1462.

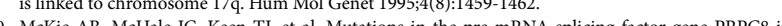
autosomal dominant retinitis pigmentosa (RP13). Hum Mol Genet 2001;10(15):1555-1562.

11. Rebello G, Ramesar R, Vorster A, et al. Apoptosis-inducing signal sequence mutation in carbonic Rebello G, Ramesar R, Vorster A, et al. Apoptosis-inducing signal sequence mutation in carbonic
anhydrase IV identified in patients with the RP17 form of retinitis pigmentosa. Proc Natl Acad Sci USA 2004;101(17):6617-6622. DOI:10.1073/pnas.0401529101

12. International Human Genome Sequencing Consortium. Finishing the euchromatic sequence of the human genome. Nature 2004;431(7011):931-945. DOI:10.1038/nature03001

13. Division of Human Genetics, University of Cape Town. Retinal Degeneration Research Project, List of Publications and Previous Postgraduate Students. http://www.humangenetics.uct.ac.za/ sites/default/files/image_tool/images/237/RDD_publications.pdf (accessed 7 February 2016).

14. Asper Ophthalmics, Asper Biotech. List of Genetic Tests for Inherited Eye Disease. http://www. asperbio.com/asper-ophthalmics (accessed 25 August 2015).

15. Roberts LJ, Nossek CA, Greenberg LJ, Ramesar RS. Stargardt macular dystrophy: Common ABCA4 mutations in South Africa establishment of a rapid genetic test and relating risk to patients. MOL Vis 2012;18:280-289.

16. Roberts L, George S, Greenberg J, Ramesar RS. A founder mutation in MYO7A underlies a significant proportion of usher syndrome in indigenous South Africans: Implications for the African diaspora. Invest Opthalmol Vis Sci 2015;56(11):6671-6678. DOI:10.1167/iovs.15-17028

17. Tõnisson N, Kurg A, Kaasik K, Lõhmussaar E, Metspalu A. Unravelling genetic data by arrayed primer extension. Clin Chem Lab Med 2000;38(2):165-170. DOI:10.1515/CCLM.2000.025

18. O'Sullivan J, Mullaney BG, Bhaskar SS, et al. A paradigm shift in the delivery of services for diagnosis of inherited retinal disease. J Med Genet 2012;49(5):322-326. DOI: 10.1136/jmedgenet-2012-100847

19. Ratnapriya R, Swaroop A. Genetic architecture of retinal and macular degenerative diseases: The promise and challenges of next-generation sequencing. Genome Med 2013;5(10):84. DOI:10.1186/ gm 488

20. Greenberg J, Roberts L, Bruwer Z, Schoeman M, Loggenberg K, Loubser F. Delivery of an ophthalmic genetic service in South Africa. SA Ophthalmol J 2010;5(2):14-19.

21. Basson F, Futter MJ, Greenberg J. Qualitative research methodology in the exploration of patients perceptions of participating in a genetic research program. Ophthalmic Genet 2007:28(3):143-149. DOI:10.1080/13816810701356627 